\title{
Forcing or Forced Exertions in Amalgamation of Nanoparticles and Particles inside the Solution
}

\author{
Mubarak Ali1, ${ }^{*}$ and I-Nan $\mathrm{Lin}^{2}$ \\ 1Department of Physics, COMSATS University Islamabad, Park Road, Islamabad- \\ 45550, PAKISTAN, *E-mail: mubarak74@mail.com, mubarak74@comsats.edu.pk \\ 2Department of Physics, Tamkang University, Tamsui Dist., New Taipei City 25137, \\ Taiwan (R.O.C.), E-mail: inanlin@mail.tku.edu.tw
}

Abstract: The study of forcing or forced exertions in amalgamating or amalgamated different featured nanoparticles and particles is important for the scientific societies. Observing live dynamics of nanoparticles and particles in the course of their amalgamation inside the solution is a challenging task. It is difficult to discuss amalgamating nanoparticles and particles only based on their visualized dynamics. However, suitably captured microscopic images of agglomerations of nanoparticles and particles can identify different modes of forcing or forced exertions. Nanoparticles and particles just at resting positions amalgamate under resultant differences of exerting forces not only related to poles of occupied quadrants, but also related to poles of their own drawn origins. In this context, colloidal entities amalgamating inside the solution from their directing sides experience a greater exertion of force. In amalgamation of nanoparticles and particles, the exerting force is mute, alert and functioning successively; contrarily, the energy behavior remains functioning, alert and mute successively. Amalgamations depend on the features and conceived focusing lengths of colloidal entities, which are through the origins of references of their exerting forces under the resultant differences. In amalgamation of nanoparticles and particles having geometrical shapes, electrons of their elongated atoms along each side deal with even exertions of force. This is not the case in nanoparticles and particles of distorted shapes. Colloidal nanoparticles and particles 
are considered the best candidates to track nature of exerting forces, and so is the case with energy behaviors, too.

Keywords: Nanoparticles; Particles; Forcing exertion; Forced exertion; Suspected point; Focusing length; Amalgamation; Agglomeration

\section{Introduction}

In order to understand the atomic behavior in different elements, a mechanism of forcing or forced exertion at electron level can be considered. The exertion of force at electron level in atoms of all elements is naturally considered in action. This will lead to the understanding of forcing and forced behaviors of nanoparticles and particles in the course of their amalgamations inside the solution. It is possible to trace dynamics of amalgamations of nanoparticles and particles through their suitably captured microscopic images.

Exertion to an atom executing confined inter-state electron dynamics involves a conservative mode of force at first stage [1]. A silicon atom under neutral behavior of electrons executes confined inter-state electron dynamics to generate photon energy shaped like a wave [2]. In electronic transfer mechanism of a gaseous carbon atom, energy and force together convert state behavior into another state behavior as explored by Ali [3]; a conversion of gas state carbon atom into a different state is through the engagement (not involvement) of a non-conservative force, which is at second place, and where non-conserved energy is involved (not engaged) at first stage. The study conducted by Ali [4] discussed that engaged forces to influence electrons of gas atoms or solid atoms are through suitable amount of supplied energy. Thus, they introduced different transitional behaviors, and then atoms of none of the elements ionize [5].

Tiny particles of different features developed through the application of energy and force under different behaviors [6]. To nucleate nanoparticles and particles of various geometrical shapes through the coalescences of tiny particles, exerting forces in the immersing format were considered vital by Ali and Lin [7]; exerted forces in the formation of tiny particles at different zones of solution surface were studied, and a source of levitational force explored. Visualizing and observing the atoms at 
work, their charge[d] dynamics were studied by the application of high-resolution microscopy $[8,9]$.

Gold particles of different shapes were developed under varying precursor concentration [10]. Morphology and structure of gold tiny particles and larger particles were controlled by varying the pulse OFF to ON ratio under bipolar mode [11]. The origin of physics and chemistry of materials was discussed, where particles of extended shapes developed at a very high rate [12]. Unprecedentedly-shaped colloidal particles were developed discussing the predictor packing of triangular shaped tiny particles [13]. A different structure in silver colloids and gold colloids emerged synthesizing for nearly identical conditions of the process [14]. In addition to this, studies referred in references 10-14 also present an initial efforts toward studying mechanisms of different forcing or forced exertions. However, this work presents the mechanism of forcing and forced exertions in amalgamating and amalgamated nanoparticles and particles under the extended version of knowledge and approach.

It is important to understand the individual dynamics of formation of tiny particles before studying assembling for a larger particle [15]. Understanding coalescence of tiny particles for a larger particle will enable to deal with atoms and molecules as materials of tomorrow [16].

The on-going research efforts should also use dynamics in addition to geometry and entropy to explain the structure [17]. Park et al. [18] introduced a method to determine three-dimensional structure of individual nanoparticles in solution as they tend to be a useful means of information. The modification of building blocks to some extent introduced a complexity in their self-assembled structures [19]. Reactiondiffusion processes at the nano- and microscales were investigated providing new means of understanding in different areas [20]. Tuma et al. [21] exploited the physics of reversible phase transition from amorphous to crystal state where that was under the reset of neuron reconfiguration of atomic structure. The optical properties of vanadium dioxide changed intensely under the change in state [22]. To configure colloidal assembly, the procedure of multiple laser beams may manipulate and assemble colloidal atoms in parallel form [23]. A detailed analysis was presented for complex nano-structures and 
colloidal nanoparticles under the description of different named forces [24]. Different interactions were considered in the formation of a variety of nanoparticles [25, 26].

An understanding in the forcing and forced behaviors while amalgamation of nanoparticles and particles will help to also understand the energy behaviors at micro, nano, atomic and electron levels. Some recent studies considered force discussing the results of an adhesive performance of graphene/shape memory polymer [27], a basic mechanism of understanding the interaction between inorganic and organic nanomaterial [28], and toxicity in different gold clusters under various concentrations [29].

Various chemical compounds and precursors are used to develop a variety of nanoparticles and particles. In different methods, the set conditions also introduce forcing exertions through medium dynamics in addition to exertions of forces of their own poles drawn from nanoparticles and particles at resting positions. They amalgamate in different zones of solution to scheme stalls or agglomerates. Their modes of amalgamation identify the new parameters which have not yet been investigated or have been investigated in lesser details. Dynamics describe their behavior at all scales; dynamics identify locally arisen forces in relation to fundamental forces. The amalgamation of nanoparticles and particles in suitable zones of solution is discussed, where they exhibit agglomerations of captured microscopic images. Here, forcing and forced exertions to nanoparticles and particles while amalgamation are discussed. The study of energy behaviors for them is worked out analogously. For this, bright field transmission microscopic images of nanoparticles and particles are chosen. The development process of such nanoparticles and particles was discussed in different studies employing pulse-based electron-photon/solution interface process [10-13].

\section{Results and Discussion}

A tiny particle of geometrical or non-geometrical shape is in the side length of a few atoms. A molecule is smaller in size or of the same size as a tiny particle. A nanoparticle has length of each side in a few tens of nanometers. So, it is bigger in size than a tiny particle. This way, a particle has a length of each side in several tens 
of nanometers. In different sizes of range, all sorts of tiny particles, nanoparticles and particles are related to colloidal entities.

On development, nanoparticles and particles fill the spaces in the solution one by one. Developing nanoparticles and particles occupy different zones inside the solution depending on their rate and time of development. Prior to amalgamating under forcing exertions, they remain at rest even for an instant; they can undergo to amalgamate. The noticeable space between them depends on several factors such as: composition, dimension of beaker, positions of source dynamics, input power, the nature of ingredients and a surrounding environment, processing time, distribution density and overall features of nanoparticles and particles, etc. Both density and distribution of nanoparticles and particles influence the rate of amalgamation. The rate of amalgamating nanoparticles and particles can be controlled by choosing their selective size and shape. Particles can collide and attach under Brownian motion, laminar or turbulent flow or relative particle setting mechanism to show their agglomeration [30].

The harmonized lateral widths of nanoparticles and particles can facilitate in filling vacant spaces under a controlled orientation of amalgamation, where they can assume a new shape of their distribution. A nanoparticle or particle of geometrical shape is influenced by a force having a directional behavior. When a similar shaped nanoparticle or particle conceived the focusing of length coming within the common vicinity, it amalgamated under the even exertion of force at electron level. Focusing of size distributions in colloidal nanocrystals was first pointed out by Peng et al. [31]. Size focusing methodology is due to the different levels of stability among differentsized nanoparticles [32]. Hannah et al. [33] demonstrated size refocusing to order phase in gold-copper nanoparticles.

Input parameters along with the set-up of developing nanoparticles and particles shown in Figures 1, 2, 3 and 4 are given in the references 10, 11, 12 and 13 respectively.

In Figure 1 (a) and (b), modes of exerting forces to nanoparticles and particles to amalgamate can be traced from the bright field transmission microscope (BF-TM) images. Nanoparticles and particles having geometrical shapes amalgamate within the common vicinities in solution under their controlled behavior of forcing exertions. 
When the distance between two nanoparticles or two particles (or between both of them) exceeds the required lengths, they do not amalgamate. If they do so, they take more time. In Figure 1 (a), agglomerations of nanoparticles in three different regions of the same zone is shown. In region (1) of Figure 1 (a), two triangle-shaped particles do not adhere side by side or face to face. In region (3) of Figure 1 (a), triangle and hexagon-shaped particles adjust side by side filling the space. The nanoparticles or particles do not conceive the focusing of lengths to amalgamate indicating that their directing sides deal with uneven exertions of forces. Hence, they do not adjust side by side or face to face.
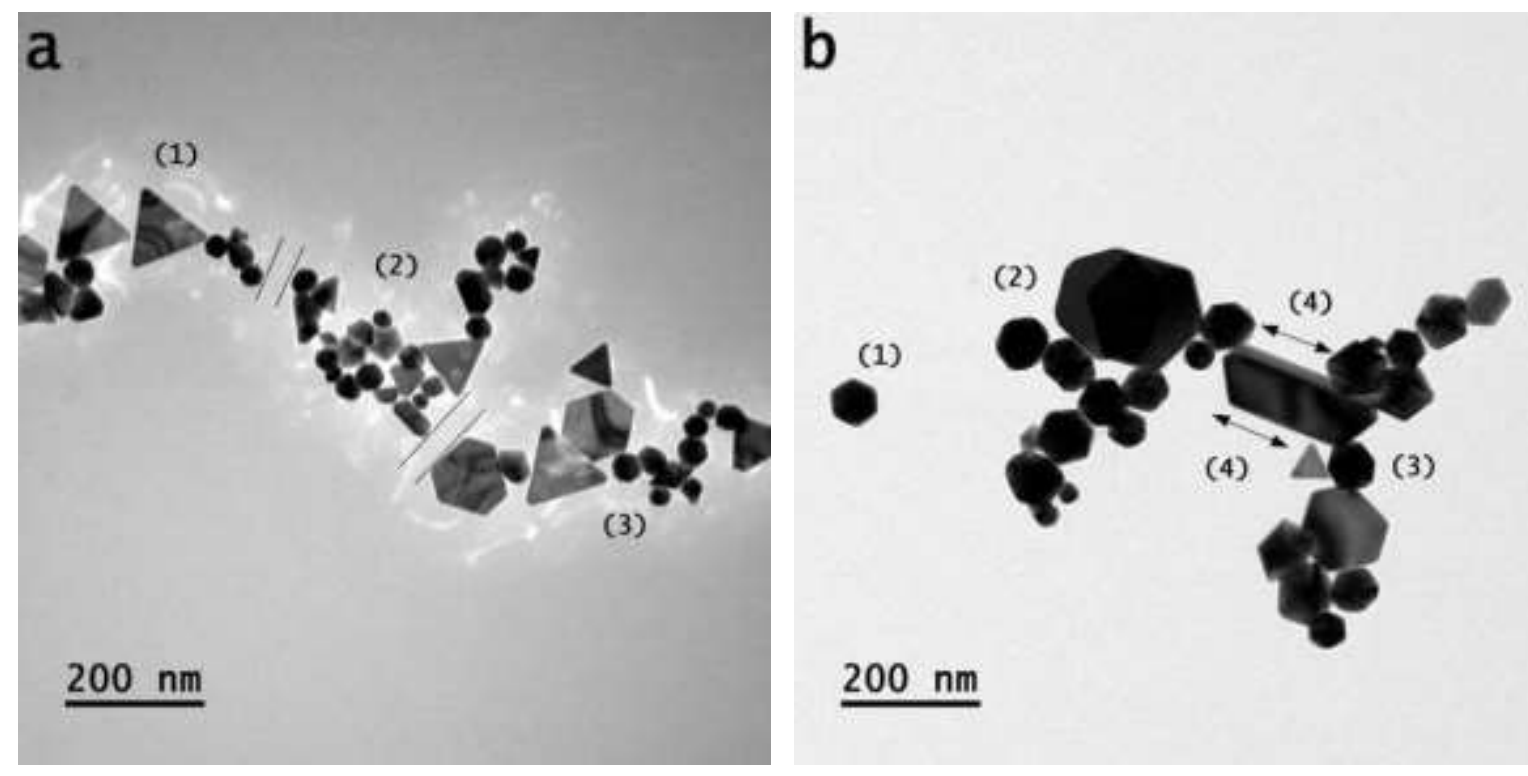

Figure 1: BF-TM images of nanoparticles and particles show modes of amalgamation in both geometrical and distorted shapes developed at (a) precursor concentration $0.10 \mathrm{mM}$, argon gas flow rate $50 \mathrm{sccm}$ and the process duration is 10 minutes, and (b) precursor concentration $0.30 \mathrm{mM}$, argon gas flow rate $100 \mathrm{sccm}$ and the process duration is 10 minutes; experimental detail is given in ref. 10

A large-sized particle labelled by (4) blocked amalgamation of nanoparticles and particles occupied regions (2) and (3) shown in Figure 1 (b). In Figure 1 (b), a nanoparticle shown in region (1) does not conceive the focusing of length to amalgamate, so it stays alone in the solution. The exertions of force to amalgamate do not synchronize. It maintains isolation till medium dynamics are not favoring to attain the length of its focus. As in Figures 1 (a) and 1 (b), nanoparticles and particles 
of different lateral widths overlapped as well, and they developed for different durations.

Different BF-TM images of amalgamated nanoparticles and particles are shown in Figure 2 (a-h). In each BF-TM image, nanoparticles and particles amalgamated by tracing a different model of forced exertions, which can be plotted. It comes with a different cluster of information not only in the physical and chemical sciences, but in the different mathematical areas, too. Their computational mathematics and physics can also be studied. When comparing the agglomerates shaped by amalgamated nanoparticles and particles in one BF-TM image to another BF-TM image, they also extract a different model of forcing exertions while amalgamating or forced exertions while amalgamated. So, they hide important science and practical usage of knowledge at multiple scales. No doubt, the given images of amalgamated nanoparticles and particles shown in this study extract different modes of forcing or forced exertions.
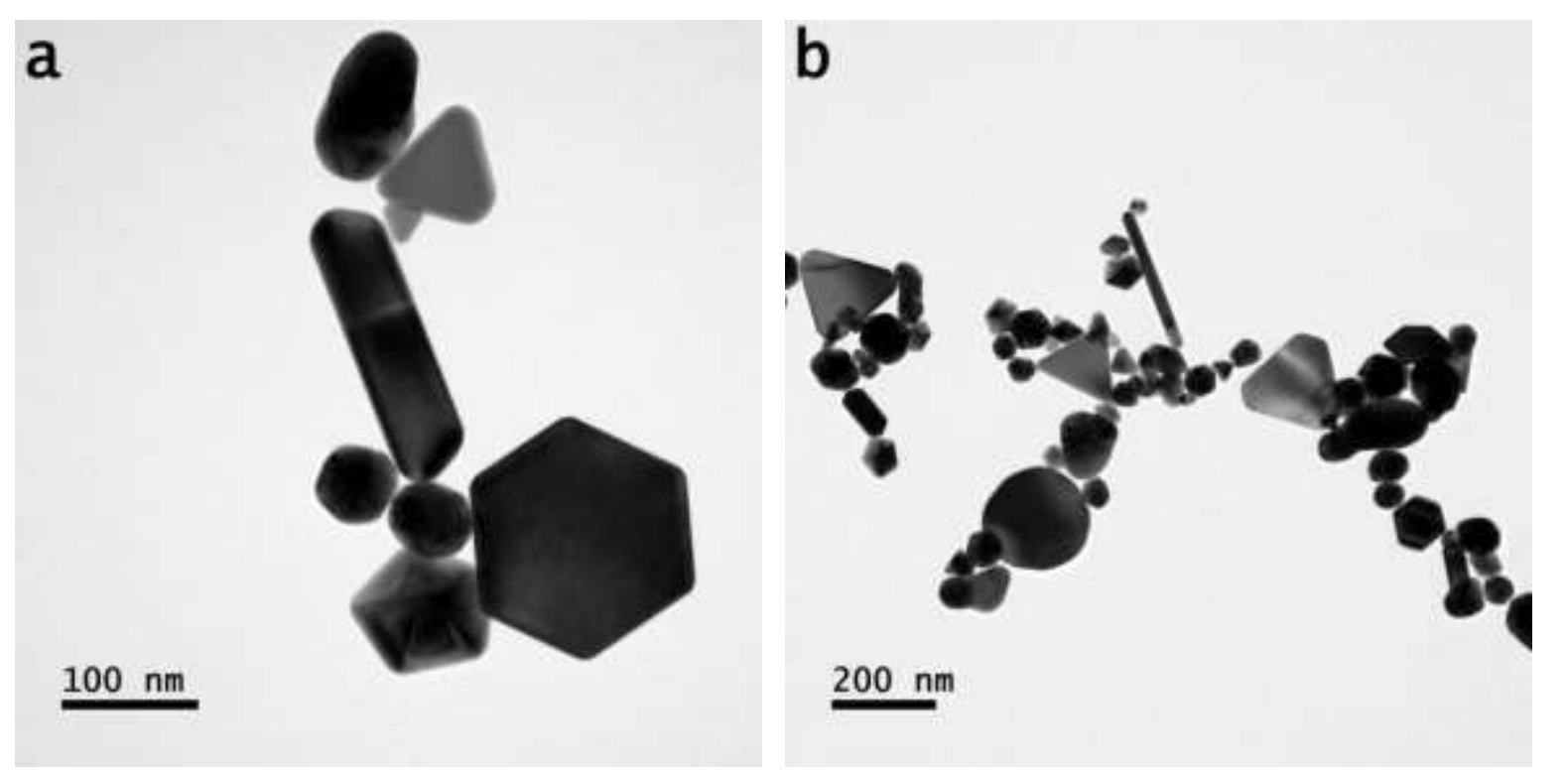


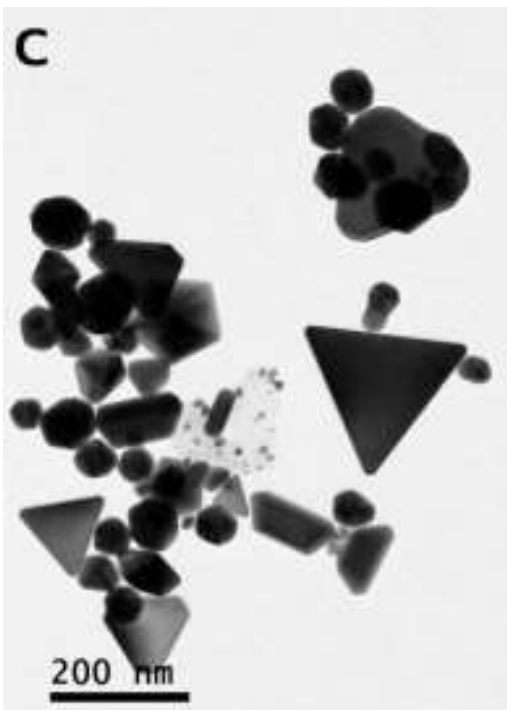

d

e

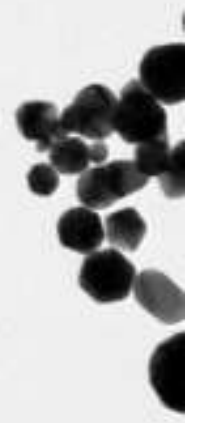

\section{$100 \mathrm{~nm}$}

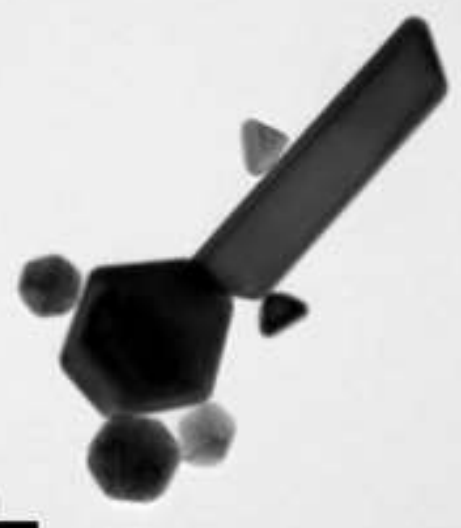

f

$100 \mathrm{~nm}$

$100 \mathrm{~nm}$ 


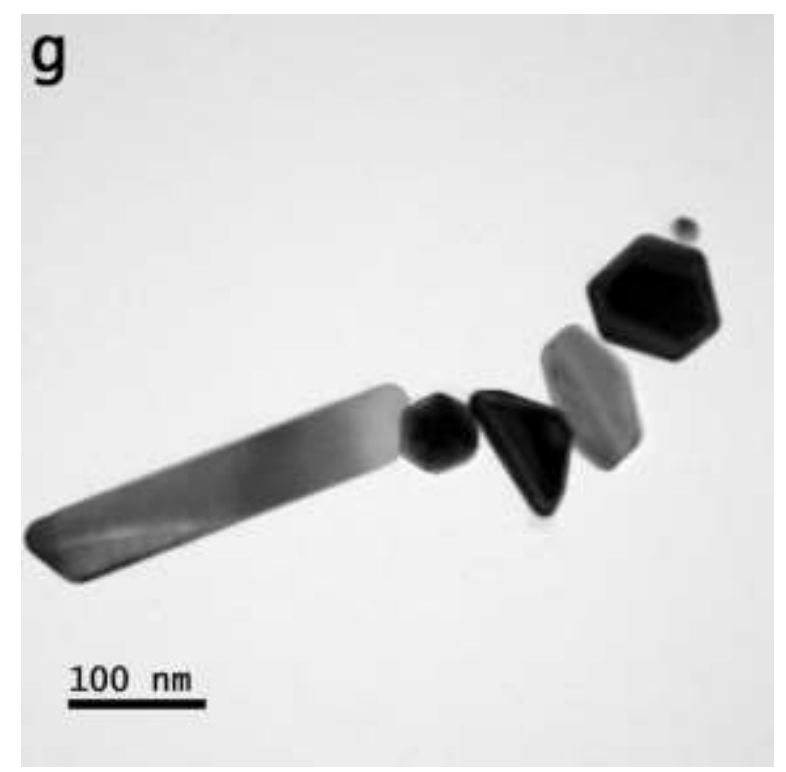

h

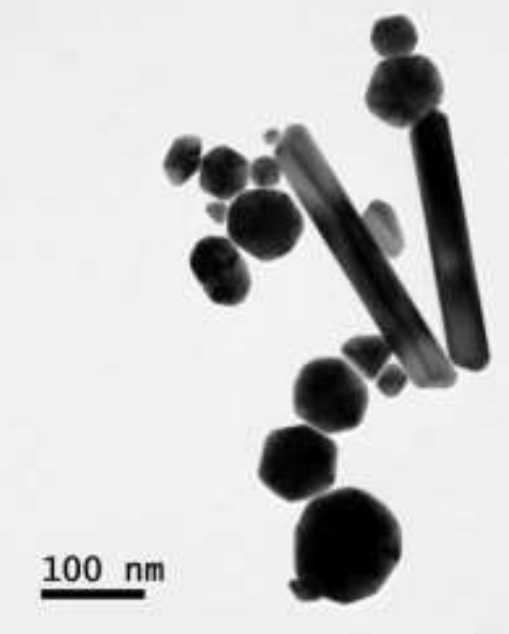

Figure 2: (a-h) BF-TM images of nanoparticles and particles show modes of amalgamation in both geometrical and distorted shapes developed at precursor concentration $0.40 \mathrm{mM}$, argon gas flow rate $100 \mathrm{sccm}$ and the process duration is 20 minutes; experimental detail is given in ref. 11

In Figure 3 (a), a hexagon-shaped particle focuses on the distorted particle with a gap of reasonable distance. They can attain favorable orientations of sides to conceive the focusing of lengths of amalgamation at common vicinity, which is through forcing exertion of the medium dynamics. When both the particles are in distorted shapes, they amalgamate under zigzag interactions shown in Figure 3 (b). Conceiving focusing of lengths for their amalgamation depends on the variation of forcing exertions. Their interactions with solution occur by plotting the different trajectories. On amalgamation under zigzag interactions, they can also deform, where they can show the scheme of distribution in different ways as given in Figure 3 (c). In different shapes of agglomerations, nanoparticles and particles in microscopic images either adhering tightly, adhering loosely or overlapping. 


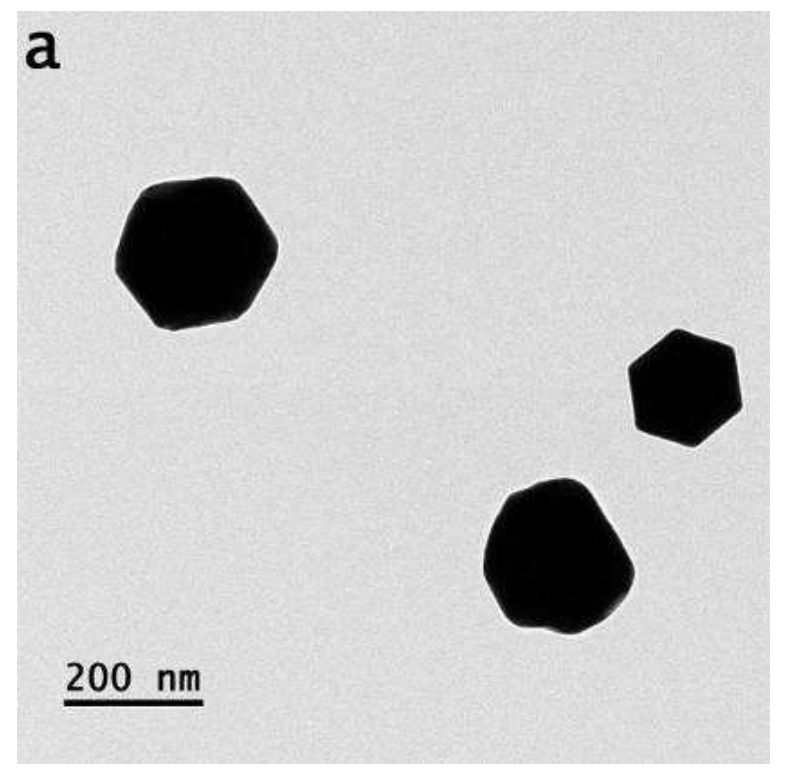

b

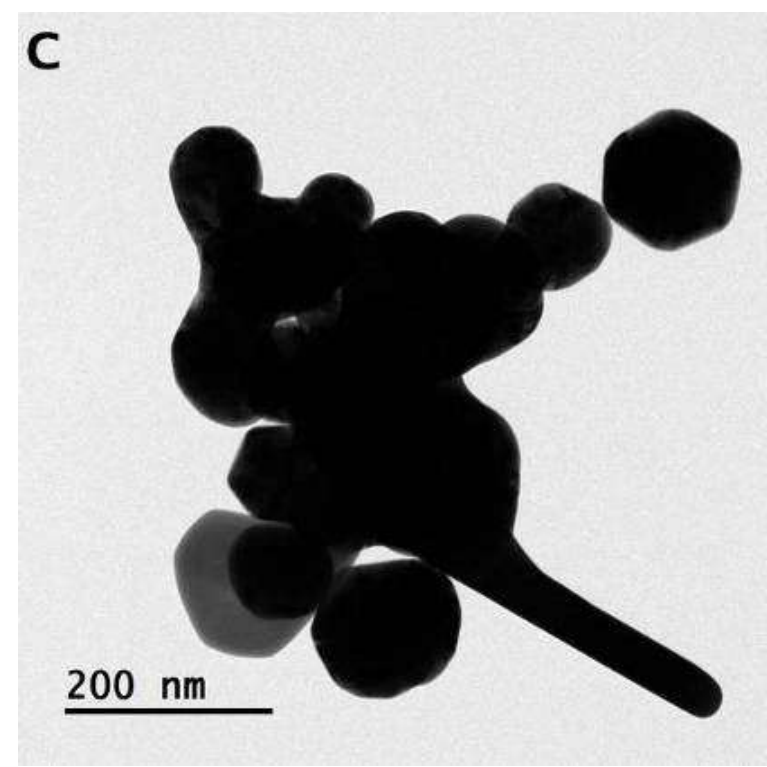

Figure 3: BF-TM images of different particles show modes of amalgamation in both geometrical and distorted shapes developed at (a) and (b) precursor concentration $0.60 \mathrm{mM}$, argon gas flow rate 100 $\mathrm{sccm}$ and the process duration is 10 minutes and (c) precursor concentration $0.60 \mathrm{mM}$, argon gas flow rate $100 \mathrm{sccm}$ and the process duration is 15 minutes; experimental detail is given in ref. 12 In Figure 4 (a-d), different microscopic images display distribution of nanoparticles and particles in different manners. Amalgamated nanoparticles and particles under forced exertions to scheme different agglomerations are shown in Figure 4 (a-d). Their predicted zones inside the solution are sketched in Figure 5. 

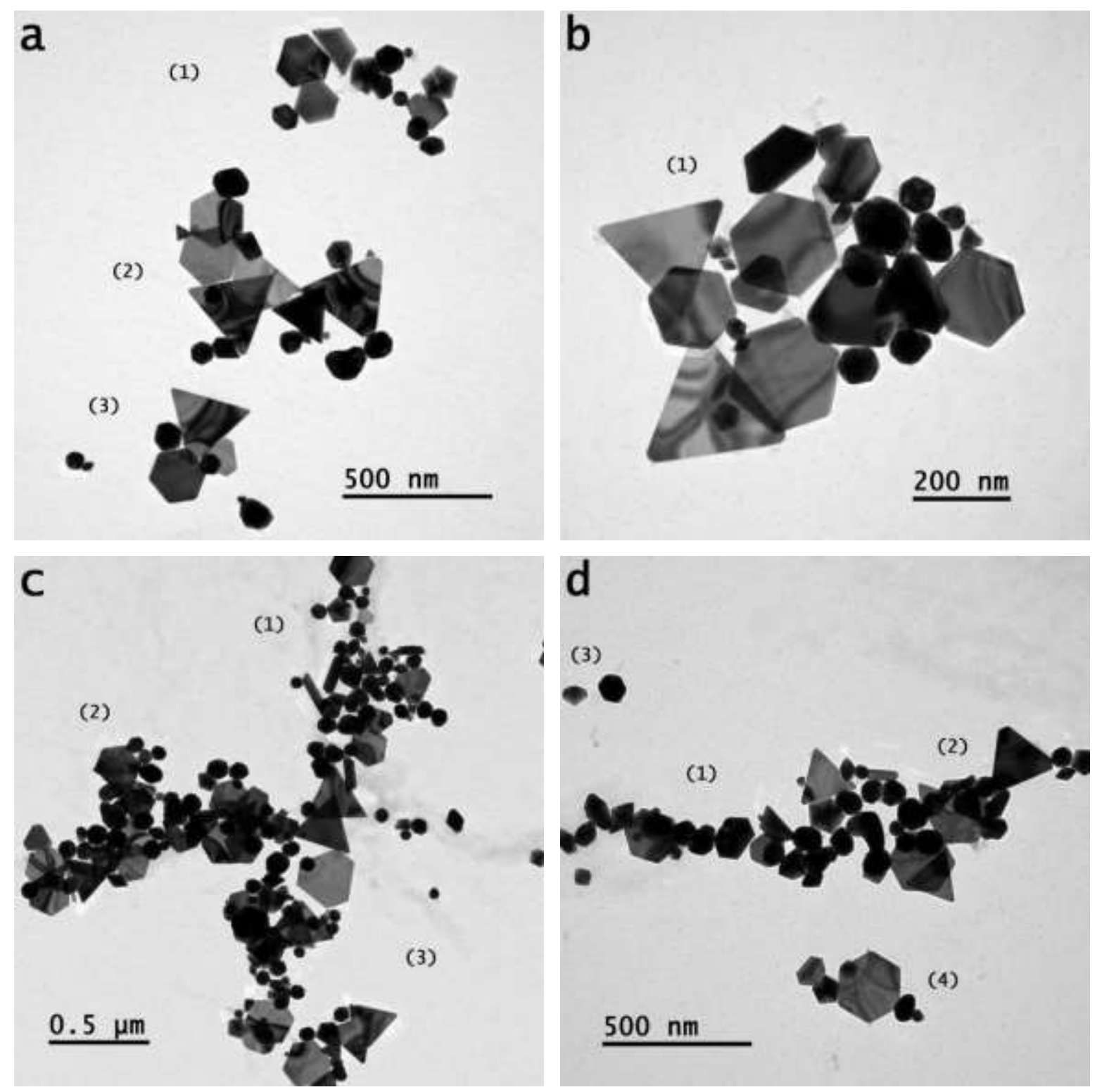

Figure 4: (a-d) BF-TM images of different nanoparticles and particles show modes of amalgamation in both geometrical and distorted shapes developed at precursor concentration $0.30 \mathrm{mM}$, argon gas flow rate $100 \mathrm{sccm}$ and the process duration is 2 minutes; experimental detail is given in ref. 13 Developed nanoparticles and particles inside the solution are categorized in terms of agglomerating in four quadrants, i.e., Q1, Q2, Q3 and Q4. These are displayed in Figure 5. The centre of four quadrants is related to a point where different nanoparticles and particles developed. Each quadrant can display more than one region of amalgamation of nanoparticles and particles. They conceive the respective focusing of lengths to amalgamate at common vicinity. The focus can be worked out by finding the force exerted along the sides of nanoparticles or particles directed to 
amalgamate them. Exertions of forces first orientate by tuning the sides directing to amalgamate their nanoparticles and particles. Nanoparticles and particles shown in Figure 4 (a) depict the centers of joining in three different regions of solution within the same zone (Q1) as labelled in Figure 5. Nanoparticles and particles shown in Figure 4 (b) depict vicinity of amalgamation in one zone only (Q2), which is shown in Figure 5. Nanoparticles and particles shown in Figure 4 (c) depict vicinities of amalgamations in different zones, i.e., Q2, Q3 and Q4 as in Figure 5. Nanoparticles and particles of Figure 4 (d) predict common vicinity of amalgamation in single zone, i.e., Q4 (along x-axis) as given in Figure 5.

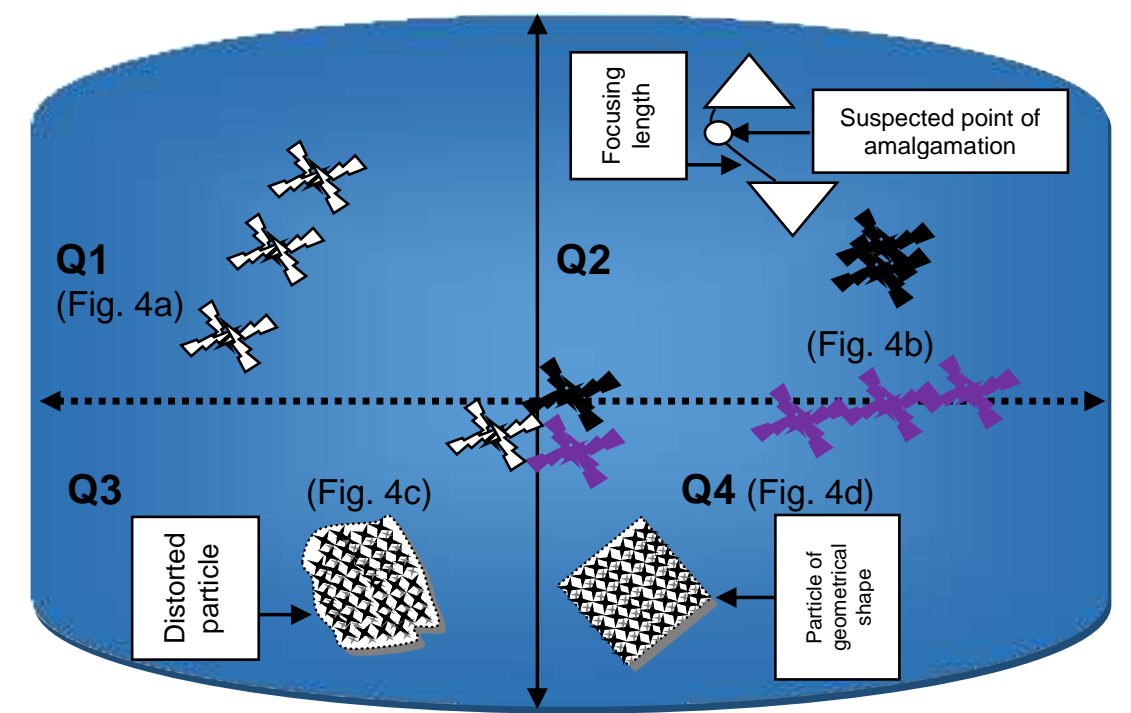

Figure 5: In Q1, Q2, Q3 and Q4, tracing forced exertions of amalgamated nanoparticles and particles is shown in Figure 4 (a-d); suspected point of amalgamation of two identical shaped particles is shown in the vicinity of Q2, a distorted particle having non-uniform lateral and adjacent orientations of electrons is shown in the vicinity of Q3 and a particle of geometrical shape dealing with uniform lateral and adjacent orientations of electrons is shown in the vicinity of Q4; a sketch is drawn in estimation

Different interactions prevail in the course of amalgamations of colloidal entities. On amalgamation in regions of suitable zones, colloidal entities design the overall shape of their distributions. The overall displayed shape of amalgamated nanoparticles and particles, or both of them under exertions of forces is called agglomeration. Nanoparticles and particles amalgamate inside the solution by locating their common vicinity. Initially, a nanoparticle or particle remains static with 
the solution. In static interaction of a nanoparticle or particle with the solution, force remains in mute mode, while energy behavior remains in functioning mode. In limiting interaction of a nanoparticle or particle with the solution, a force remains in alert (in ready to work, apply, influence or exert) mode, while energy also remains in alert (but in ready to quiet, not work, not apply, not influence or not exert) mode. In kinetic interaction of a nanoparticle or particle with the solution, force remained in functioning (working, applying, influencing or exerting) mode, while energy remained in mute (quiet) mode.

On amalgamation of colloidal entities in suitable vicinity of solution, their forced exertions can be influenced by reflexes of surrounding environment, where their energy functions either to keep them still or to keep them in collective motion.

A kinetic interaction of a nanoparticle or particle having geometrical shape with solution is a controlled interaction. A focusing of length of nanoparticle or particle in geometrical shape with respect to a focusing of length of another identically featured nanoparticle or particle does not infer their amalgamation at midpoint (half-length) of distance noticeable between them. Nonetheless they locate a suspected point of amalgamation, which is at a certain distance of their conceived focusing of lengths. They will coordinate to amalgamate (at a suspected point) with reference to exerting force along directing sides. Consequently, a suspected point of amalgamation of two nanoparticles or particles is located through the resultant differences of poles' forces in occupied quadrant and their own poles drawn at resting positions shown in estimation in Q2 of Figure 5.

Each particle of geometrical shape conceives its own length of focusing but with reference to the attending one(s) at common vicinity. This is due to the coordination of other particle(s) of geometrical shape(s). So, nanoparticles and particles of geometrical shapes amalgamate at common vicinities under the resultant differences of forces of origins in occupied quadrants and resultant differences of forces of their own drawn origins. Experiencing the differences of forces of their own poles drawn, they simultaneously deal with the differences of forces of poles drawn in occupied quadrants in the amalgamation of nanoparticles and particles of geometrical shapes. Their amalgamation is at least under two origins drawn: internal origin depicted by their own poles drawn at resting position, and external origin depicted by the 
occupied region in quadrant. This way, they consider the resultant differences of forces belonging to at least two origins in their amalgamation. In this manner, the focusing lengths of amalgamating nanoparticles and particles are directly proportional to the resultant differences of exerting forces obtained along their directing sides. The introduction of a suitable constant equalizes the proportionality between two variables. A force of the quadrant as per traced poles is already there to exert. A nanoparticle (or particle) occupies a certain region of the quadrant. It also traces its own poles to deal with the forcing exertions. So, in the case of considering plain region of nanoparticles or particles having identical geometrical shapes, they amalgamate side by side inside the solution by coordinating with the forces of two origins mainly. There can be the influence of third origin of reference or even more origins of references when nanoparticles or particles are neither in geometrical shapes nor in matched lateral width.

To calculate the resultant difference of an exerting force to nanoparticle or particle of geometrical shape from two origins, a transformational law under the rotation of basis vectors can be helpful. There can be more parameters (or factors) involved when studying the forcing or forced exertions in amalgamations of nanoparticles and particles. This can be mainly related to features of nanoparticles and particles along with their occupied vicinities inside the solution. In order to establish the concrete equations on amalgamations of different featured nanoparticles and particles inside the solution, much work is required. Different lateral widths of nanoparticles or particles with the same shape can also alter the modes of their amalgamation. In this context, both surface topography and aspect ratio of nanoparticles and particles contribute in the study of modes of their amalgamations. In the case of distorted nanoparticles or particles, a kinetic interaction with solution is a largely uncontrolled interaction. When nanoparticles or particles possess distorted shapes, their static interaction, limiting interaction and kinetic interaction with the solution occur in more chaotic ways, where they can further deform their shapes.

Normally, different frictional behaviors are being considered in the case of solid surfaces when in contact. However, in a colloidal solution, a partial behavior of the force exists, which leads to introduce a partial behavior of friction in the form of interaction. So, nanoparticles and particles deal with their partial interactions with the 
solution. Entered forces deal with even exertion throughout the solution to keep liquidness.

In distorted nanoparticles and particles, both adjacent and lateral orientations of electrons deal with non-uniform orientations in their atoms. Non-uniform orientations of electrons keep irregular ordering of atoms in the development of nanoparticles and particles. Availability of a deformed atom in the development of a nanoparticle or particle means a distorted nanoparticle or particle. Such nanoparticle or particle does not draw a straight trajectory of appreciable length when conceiving its length of focusing. It was found in the selective area patterns that the electrons belonging to central rings along with outer rings of elongated atoms in hexagon and triangleshaped particles remained in lateral orientations, but electrons belonging to only central rings of atoms in rod and bar-shaped particles remained in lateral orientations [7]. Moreover, hexagon and triangle-shaped particles are related to multi-dimensional (MD) particles, whereas rod and bar-shaped particles are related to one-dimensional (1D) particles. A difference between the adjacent orientation and lateral orientation was disclosed by Ali and Lin [12]; however, a detailed study regarding this was presented separately [7].

Therein, the nanoparticles and particles have distorted shapes. Their atoms attain the uneven orientations of electrons. Their electrons neither orientate laterally nor adjacently. Thus, conceived focusing lengths of such nanoparticles and particles result in the non-uniform behaviors of amalgamations (Q3 in Figure 5). Electrons of surface atoms in amalgamating nanoparticles and particles are, in fact, nonorientationally based. For nanoparticles and particles having geometrical shapes, atoms keep the orientations of electrons laterally and adjacently for each face. So, they amalgamate under controlled angle interactions (Q4 in Figure 5). Electrons of surface atoms keep positioned orientationally in their amalgamating particles.

In each side of nanoparticle or particle having geometrical shape, electrons (of elongated atoms of arrays which formed structures of smooth elements) positioned orientationally. In all structures of smooth elements forming each mono layer (not monolayer) of nanoparticle or particle, electrons of elongated atoms (forming each face) orientated along with each side as shown in Q4 of Figure 5. The configurations of electrons of elongated atoms in each face of $1 \mathrm{D}$ or MD nanoparticle or particle 
remain uniformly orientated. Hence, the gained (or incurred) orientational positions of electrons on each face of such nanoparticles or particles deal with a structure of consistent inter-state gap. According to the suitable design of a material, a structure of consistent inter-state electron gap can be made to work in 1D, 2D or 3D. Such kinds of materials (nanoparticles and particles) deal with an accelerated propagation of photons. As inter-state electron gaps of elongated atoms in nanoparticles or particles of $1 \mathrm{D}$ or MD shapes allow photons to carry forcing energy, so their path (inter-state electron gap) can be referred to as photonic band gap. To a large extent, this is not the case with distorted nanoparticle or particle as their electrons nonuniformly orientated to a large extent. Thus, they locally dissipate heat energy in water to work as catalytic agents. Further details about inter-state electron gap replaced instead of band gap in materials of conductor, semi-conductor or insulator behavior are given in a separate study [5].

Both solution composition and medium source influence the forcing or forced exertions in amalgamation of nanoparticles and particles. Thus, trajectories of suspected points of amalgamations on conceived lengths of focusing in different featured nanoparticles and particles can be plotted for several ways. These require indepth studies to explore the underlying sciences. Here, different features of nanoparticles and particles need to be considered. These studies may explore interesting new insights for the scientific community. Modes of forcing or forced exertions to nanoparticles and particles of different features are needed to be explored. A behavior of force to distorted particle and particle of geometrical shape can be selected in different ways. Here both computational physics and mathematics can be helpful to trace the forces exerting along their directing sides. These open new areas for researching and applying new knowledge at multiple scales.

In mathematics, new knowledge in the fields of vector analysis, vector space, topology, mechanics, calculus and analytical geometry, etc. can be established. As the shape of nanoparticles and particles becomes more and more distorted, the influence of forces in different formats also becomes more and more chaotic. This is because neither the precise length nor the precise diameter of these particles can be measured. To measure their approximate size, it is required to locate their centre of 
mass first. This way, the studies of colloids provide vast room for conducting new research.

In amalgamation of nanoparticles and particles, the relation of exerting forces to focusing lengths and energy behavior at different levels can open countless avenues for scientific research. Such fields of studies can also engage students, researchers and scientists to conduct different statistical analyses. Fundamental questions on the stability of colloidal nanoparticles and roles of different surfactants and ligands for them are yet to be explored [34]. A study of orientation is a new entrance to study the structure of nanoparticles and particles either in colloidal form or in other form [35].

\section{Conclusion}

Nanoparticles and particles which do not possess geometrical shapes amalgamate inside the solution in zigzag manners. Here, forcing exertions to electrons of their deformed atoms remain uneven along the sides. Their focusing lengths plot complex trajectories having no significant lengths; they approach, they adhere, they overlap and they amalgamate under various static, limiting and kinetic interactions. They can deform further while conceiving focusing lengths under changing kinetic modes.

Nanoparticles and particles that possess geometrical shapes amalgamate under controlled exertions of force as electrons of elongated atoms get orientated uniformly along the sides. Nanoparticles and particles of geometrical shapes amalgamate under the differences of exerting forces originating from mainly two origins of references. On amalgamation, such two nanoparticles or particles can join side by side or face to face at midpoint of noticeable distance (length) between them. Here in the ideal condition, exerting forces along directing sides of amalgamation are in the balanced and controlled manners. To amalgamate a nanoparticle or particle of geometrical shape, a conceived focusing length becomes functioning from the side having increased even exertion of force. To amalgamate, focusing lengths of such nanoparticles or particles plot trajectories in directional manners having significant lengths. They undertake standard interactions to amalgamate inside the solution, where forcing or forced exertions to electrons of elongated atoms are worked in a diligent manner by successively remaining mute (in static behavior), alert (in limiting 
behavior) and functioning (in kinetic behavior). Hence, a forcing or forced exertion to nanoparticle or particle originates energy behavior accordingly.

To amalgamate nanoparticles or particles at common vicinity, in addition to resultant differences of exerting forces of poles in occupied quadrants of medium, they also deal with the resultant differences of forces of their own poles drawn at resting positions. So, a conceived focusing length of a nanoparticle or particle functions under the resultant difference of force of medium poles (origin) together with its own poles (origin) drawn. A force resulted along the directing side of a particle at resting position is due to the resultant difference of forces of medium poles and their own poles drawn, which will lead to its amalgamation at a located suspected point by conceived length of focusing.

Nanoparticles and particles of perfect geometrical shapes having the same lateral lengths amalgamate side by side, where they deal with quantized exertions of force, so they also deal with energy behavior in quantized manner. They amalgamate for established orientations located through their conceived focusing of lengths, so they show symmetry in amalgamation. This is also the case in microscopic images of colloidal entities as they foresee forcing exertions for amalgamations.

In nanoparticles and particles of geometrical shapes, electrons of atoms deal with uniform orientation along each side of the shape. Electrons experience the even forcing exertions along the poles, where they remain clamped by their energy knots. In elongated atoms, energy knots forming filled and unfilled states of electrons do not experience the forcing exertions. However, energy knots are influenced uniformly by the varied force (orientation) and energy (potential) of their electrons. Distorted nanoparticles and particles deform in their shapes, where they do not form sides of established lengths. Thus, they do not have the orientated electrons of the same orientation along the sides.

Nanoparticles and particles of selective features amalgamate under controlled applications of forced exertions. They can be used effectively as medication for human life. Like plants, too serve as medication for environment. The studies of forcing or forced exertions to nanoparticles and particles inside the solution help develop sustainable science and technology covering applications of broad range in 
catalysis, electro-optics, biomedical, energy and diversified eco-friendly environments. Hence, they open several new routes for research and exploration.

\section{Experimental details}

Different BF-TM images discussed in Figures 1, 2, $3 \& 4$ are synthesized by setting different process parameters, where the main parameters are given in the captions of the figures. Layouts of the setup along with different conditions are given in earlier references [10-13]. The method employed to synthesize nanoparticles and particles discussed in this work can be referred to as photochemical method, or conventionally an electrochemical method in chemical sciences. It is also called pulse-based electron-photon/solution interface process in the studies references 10 to 13 .

\section{Acknowledgements}

Mubarak Ali sincerely thanks the National Science Council (now Ministry of Science and Technology) Taiwan (R.O.C.) for awarding postdoctorship: NSC-102-2811-M032-008 (August 2013- July 2014). Authors thank Mr. Chien-Jui Yeh for helping in transmission microscope operation.

\section{References:}

[1] Ali, M. Structure Evolution in Atoms of Those Elements Executing Confined Inter-State Electron Dynamics. https://arxiv.org/abs/1611.01255v22, 2020.

[2] Ali, M. Heat and Photon Energy Phenomena: Dealing with Matter at Atomic and Electronic Level. https://www.preprints.org/manuscript/201701.0028/v10, 2017.

[3] Ali, M. Atomic Structure and Binding of Carbon Atoms. https://www.preprints.org/manuscript/201801.0036/v10, 2019.

[4] Ali, M. Atoms in Gaseous and Solid States. https://www.researchgate.net/publication/323723379, 2020.

[5] Ali, M. Atoms of None of the Elements Ionize While Atoms of Inert Behavior Split by Photonic Current. https://arxiv.org/abs/1611.05392v23, 2020. 
[6] Ali, M. Tiny-Shaped Particles Developing Mono Layer Shape Dealing with Localized Gravity in Solution Surface. https://arxiv.org/abs/1609.08047v23, 2020.

[7] Ali, M. \& Lin, I-N. Nucleation and Structural Identification in Gold Particles of High Aspect Ratios Developed through Mechanistic Approach. https://doi.org/10.26434/chemrxiv.11475300.v2, 2020.

[8] Zhu, Y., Ramasse, Q. M., Brorson, M., Moses, P. G., Hansen, L. P., Kisielowski, C. F. \& Helveg, S. Visualizing the Stoichiometry of Industrial-Style Co-Mo-S Catalysts with Single-Atom Sensitivity. Angew. Chem. Int. Ed. 2014, 53, 10723-10727.

[9] C. Kisielowski, Observing Atoms at Work by Controlling Beam-Sample Interactions. Adv. Mater. 2015, 27, 5838-5844.

[10] Ali, M. \& Lin, I -N. Development of Gold Tiny Particles and Particles in Different Sizes at Varying Precursor Concentration. Adv. Nat. Sci.: Nanosci. Nanotechnol. 2020, 11, 015006.

[11] Ali, M. \& Lin, I $-\mathrm{N}$. Controlling morphology-structure of gold tiny particles, nanoparticles and particles at different pulse rates and pulse polarity. $A d v$. Nat. Sci.: Nanosci. Nanotechnol. 2019, 10, 025015.

[12] Ali, M. \& Lin, I - N. Formation of tiny particles and their extended shapes: origin of physics and chemistry of materials. Appl. Nanosci. 2019, 9, 13671382.

[13] Ali, M., Lin, I - N. \& Yeh, C. -J. Predictor Packing in Developing Unprecedented Shaped Colloidal Particles. NANO 2018, 13, 1850109.

[14] Ali, M., Lin, I-N. \& Yeh, C. -J. Tapping Opportunity of Tiny-Shaped Particles and Role of Precursor in Developing Shaped Particles. NANO 2018, 13, 1850073.

[15] Link, S. \& El-Sayed, M. A. Shape and size dependence of radiative, nonradiative and photothermal properties of gold nanocrystals. Int. Rev. Phys. Chem. 2000, 19, 409-453.

[16] Glotzer, S. C. \& Solomon, M. J. Anisotropy of building blocks and their assembly into complex structures. Nature Mater. 2007, 6, 557-562. 
[17] Manoharan, V. N. Colloidal matter: Packing, geometry, and entropy. Science 2015, 349, 1253751.

[18] Park, J. et al. 3D structure of individual nanocrystals in solution by electron microscopy. Science 2015, 349, 290-295.

[19] Evers, C. H. J., Luiken, J. A., Bolhuis, P. G. \& Kegel, W. K. Self-assembly of microcapsules via colloidal bond hybridization and anisotropy. Nature 2016, 534, 364-368.

[20] Epstein, I. R. \& Xu, B. Reaction-diffusion processes at the nano- and microscales. Nature Nanotech. 2016, 11, 312-319.

[21] Tuma, T., Pantazi, A., Gallo, M. L. \& Eleftheriou, E. Stochastic phase-change neurons. Nature Nanotech. 2016, 11, 693-699.

[22] Rensberg, J. et al. Active Optical Metasurfaces Based on Defect-Engineered Phase-Transition Materials. Nano Lett. 2016, 16, 1050-1055.

[23] Lin, L. et al. Opto-thermophoretic assembly of colloidal matter. Sci. adv. 2017, 3, e1700458.

[24] Gargiulo, J. et al. Understanding and Reducing Photothermal Forces for the Fabrication of Au Nanoparticle Dimers by Optical Printing. Nano Lett. 2017, 17, 5747-5755.

[25] Figliozzi, P. et al. Driven optical matter: Dynamics of electrodynamically coupled nanoparticles in an optical ring vortex. Phys. Rev. E 2017, 95, 022604.

[26] Nan, F., Han, F., Scherer, N. F. \& Yan, Z. Dissipative Self-Assembly of Anisotropic Nanoparticle Chains with Combined Electrodynamic and Electrostatic Interactions. Adv. Mater. 2018, 30, 1803238.

[27] D. Tan, et al. Switchable Adhesion of Micropillar Adhesive on Rough Surfaces, Small 2019, https://doi.org/10.1002/smll.201904248.

[28] A. K. Agrawal, P. K. Sahu, S. Seth, M. Sarkar. Electrostatically Driven Forster Resonance Energy Transfer between a Fluorescent Metal Nanoparticle and JAggregate in an Inorganic-Organic Nanohybrid Material. J. Phys. Chem. C 2019, 123, 3836-3847. 
[29] K. Sokołowska, et al. Towards Controlled Synthesis of Water-Soluble Gold Nanoclusters: Synthesis and Analysis. J. Phys. Chem. C 2019, 123, 26022612.

[30] Kramer, H. J. M. \& van Rosmalen, G. M. in Enclyclopedia of Separation Science, Overview: Crystallization (Editor-in-Chief: Ian D. Wilson), 2000, 6484, Elsevier Science Ltd.

[31] Peng, X. G., Wickham, J. \& Alivisatos, A. P. Kinetics of II-VI and III-V colloidal semiconductor nanocrystal growth: "Focusing" of size distributions. J. Am. Chem. Soc. 1998, 120, 5343-5344.

[32] Jin, R., Qian, H., Wu, Z., Zhu, Y., Zhu, M., Mohanty, A. \& Garg, N. Size Focusing: A Methodology for Synthesizing Atomically Precise Gold Nanoclusters. J. Phys. Chem. Lett. 2010, 1, 2903-2910.

[33] H. M. Ashberry, J. T. L. Gamler, R. R. Unocic, S. E. Skrabalak. Disorder-toOrder Transition Mediated by Size Refocusing: A Route toward Monodisperse Intermetallic Nanoparticles. Nano Letters 2019, 19, 6418-6423.

[34] Cuffari, B. Does the Structure of Gold Nanoparticles Change with Different Dopants? (2019), https://www.azonano.com/article.aspx?ArticlelD=5142

[35] Ali, M. Transition Energy, Orientation Force and Work Done in Transitional Behavior Atoms: Formulating New Principles in Thermodynamics. (2020), ChemRxiv. Preprint. https://doi.org/10.26434/chemrxiv.11553057.v3

\section{Nomenclatures/definitions:}

Dynamics - Changing aspects of different entities (atoms, tiny particles, tiny clusters, tiny grains, nano crystal, molecule like structures, nanoparticles and particles, crystallites, etc.)

Grammatical use of dynamics - Dynamics should be used as a 'noun' but mainly in 'plural' sense. Usually, an entity should consider more than one factor simultaneously occurring to change its aspect. So, dynamics are related to changing aspects of an entity. However, it can also be used in 'singular' sense, where the employed 'verb' in the sentence distinguishing its meaning

Visualized dynamics - Observation of changing aspects of different entities while recording their behavior in the solution live

Medium dynamics - Changing behavior (aspect) of the medium (solution) through the development of different entities, interactions of entities with each other, interaction of different 
entities with the walls of beaker or container, entering forced energy electrons, electron streams and atoms, entering photons of different features and absorption of heat energy in the solution, etc. A medium dynamics governs its process of synergy

Amalgamation - This is a process taken or followed by nanoparticles and particles to join, overlap or adhere inside the solution through competing actions of forces along their sides. However, tiny particles also amalgamate to develop a nanoparticle or particle at solution surface or inside the solution

Agglomeration - A zone inside the solution, where nanoparticles and particles amalgamated through a suitable mechanism of forced exertion exhibiting their overall picture or stall (of joining, overlapping or adhering)

Forcing exertion - A force disturbs to amalgamate a nanoparticle or particle inside the solution

Forced exertion - A force disturbed to amalgamate a nanoparticle or particle inside the solution

Focusing length - A focusing length of nanoparticle or particle inside the solution is conceived by locating a suspected point of its amalgamation under noticeable length (distance), where one nanoparticle (or particle) can experience (deal) force (or energy) with respect to the force (or energy) of other (nearby) nanoparticle (or particle). So, through the action of force, and in rare cases, through the action of energy, they (nanoparticles or particles or both) attempt to disturb by suspecting their amalgamation at common vicinity inside the solution. Focusing length is not useful while considering tiny particles or atoms as they do not remain still (even for a while) alone in the solution

Suspected point - It is a point located by two entities (nanoparticles or particles) prior to amalgamating at common vicinity inside the solution, which is through conceiving of lengths of focusing of two or more nanoparticles or particles. In the case of two identical shaped (and sized) nanoparticles or particles, their suspected point can be located at midpoint of noticeable distance (length) between them (a suspected point of amalgamation of two nanoparticles or particles is located under the resultant difference of forces of their own poles drawn and poles drawn in occupied quadrant of medium at resting positions in that quadrant)

Zones of solution surface - $A$ solution surface in a beaker has four zones because of the formation of four quadrants from the point of development of nanoparticles and particles

Regions of solution surface - A zone of solution surface can have one region or more than one region inside the solution to amalgamate nanoparticles or particles

Distorted nanoparticles and particles - Nanoparticles and particles having their irregular shapes, where no certain geometry of the shape is perceptible

Geometric shapes - Shapes of nanoparticles and particles having their directional behavior, which mainly develop in suitable geometry of one-dimensional or multi-dimensional shapes 
Uniform adjacent orientations of electrons - In nanoparticles or particles of geometrical shapes, electrons of outer rings (belonging to elongated atoms) deal with their uniform orientations as they are in adjacent orientations under the even exertions of force

Uniform lateral orientations of electrons - In nanoparticles or particles of geometrical shapes, electrons of inner or central rings (belonging to elongated atoms) deal with their uniform orientations as they are in lateral orientations under the even exertions of force

Non-uniform adjacent orientations of electrons - In deformed (or partially elongated) atoms of distorted nanoparticles or particles of irregular shapes, electrons of outer rings were in their non-uniform orientations as they do not possess fully adjacent orientations under uneven exertions of force

Non-uniform lateral orientations of electrons - In deformed (or partially elongated) atoms of distorted nanoparticles or particles of irregular shapes, electrons of inner or central rings were in their non-uniform orientations as they do not possess fully lateral orientations under uneven exertions of force

Uniformly orientated electrons - Electrons orientated either adjacently or laterally under the even exertion of force (s) along the poles

Non-uniformly orientated electrons - Electrons orientated either adjacently or laterally under the uneven exertion of force (s) along the poles

Electrons of outer ring(s) - In the structure of atoms belonging to different elements as referred in references 1-5 in elongated atoms of nanoparticles and particles having geometrical shapes, electrons of their outer ring(s) orientate adjacently through the engagement of even exertions of forces along the relevant poles

Electrons of central ring(s) - In the structure of atoms belonging to different elements as referred in references 1-5 in elongated atoms of nanoparticles and particles having geometrical shapes, electrons of their central ring(s) orientate laterally through the engagement of even exertions of forces along the relevant poles

Even exertion of force - In the atom, an electron clamped by its energy knot deals with even exertion of force when it undertakes uniform adjacent or lateral orientation

Uneven exertion of force - In the atom, an electron clamped by its energy knot deals with uneven exertion of force when it undertakes non-uniform adjacent or lateral orientation 


\section{Authors' biography:}

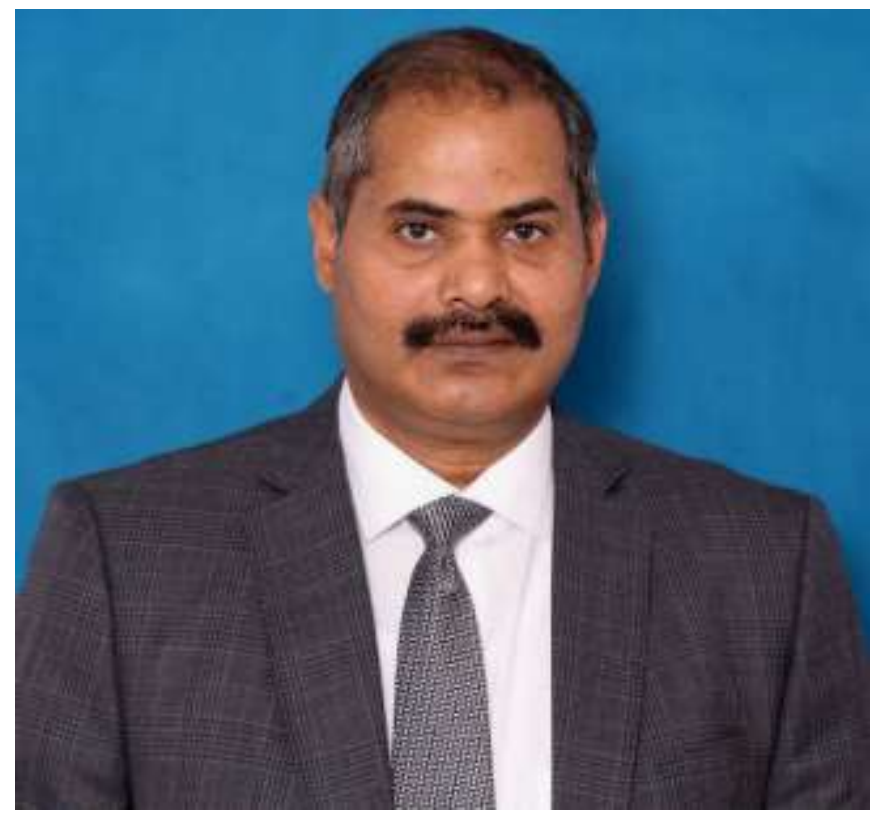

Mubarak Ali graduated from University of the Punjab with BSc (Phys \& Maths) in 1996 and MSc Materials Science with distinction from Bahauddin Zakariya University, Multan, Pakistan (1998); his thesis work completed at Quaid-i-Azam University Islamabad. He gained PhD in Mechanical Engineering from the Universiti Teknologi Malaysia under the award of Malaysian Technical Cooperation Programme (MTCP;2004-07) and postdoc in advanced surface technologies at Istanbul Technical University under the foreign fellowship of The Scientific and Technological Research Council of Turkey (TÜBITAK, 2010). He completed another postdoc in the field of nanotechnology at the Tamkang University Taipei (2013-2014) sponsored by National Science Council now M/o Science and Technology, Taiwan (R.O.C.). Presently, he is working as Assistant Professor on tenure track at COMSATS University Islamabad (previously known as COMSATS Institute of Information Technology), Islamabad, Pakistan (since May 2008) and prior to that worked as assistant director/deputy director at M/o Science \& Technology (Pakistan Council of Renewable Energy Technologies, Islamabad, 2000-2008). He was invited by Institute for Materials Research, Tohoku University, Japan to deliver scientific talk. He gave several scientific talks in various countries. His core area of research includes materials science, physics \& nanotechnology. He was also offered the merit scholarship for the PhD study by the Higher Education Commission, Government of Pakistan, but he did not avail himself of the opportunity. He also earned Diploma (in English language) and Certificate (in Japanese language) in 2000 and 2001 respectively, in part-time from the National University of Modern Languages, Islamabad. $\mathrm{He}$ is the author of several articles available at following links; https://scholar.google.com.pk/citations?hl=en\&user=UYjvhDwAAAAJ, 


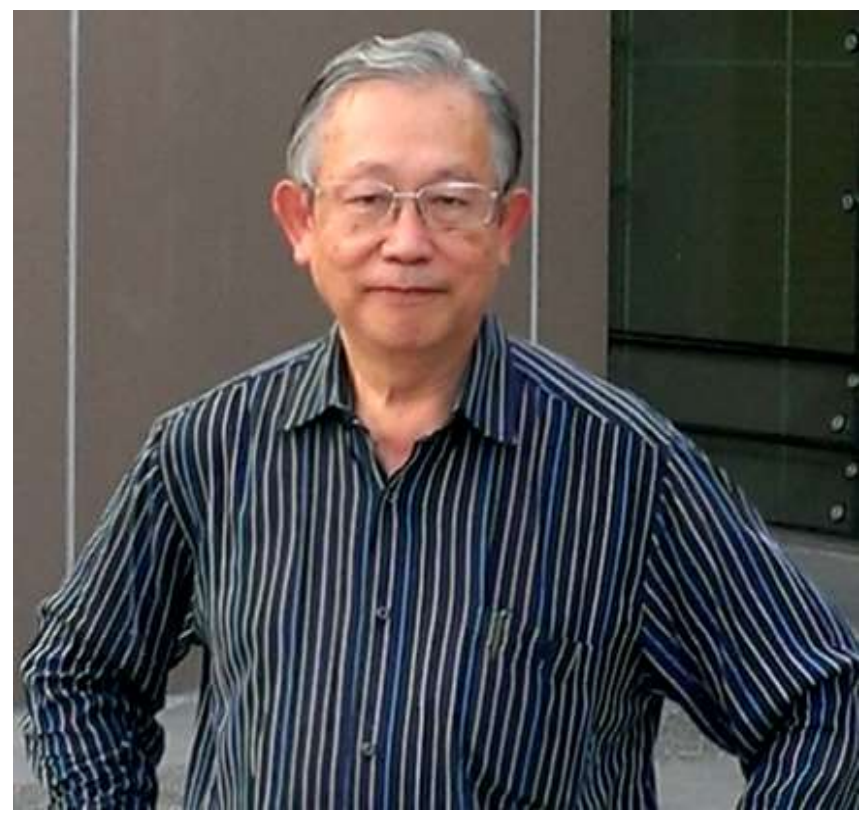

His Excellency, I-Nan Lin, worked as a Senior Professor for several years at Tamkang University, Taiwan, and now Professor Emeritus. His Excellency received the bachelor's degree in physics from National Taiwan Normal University, Taiwan, M.S. from National Tsing-Hua University, Taiwan, and the Ph.D. degree in Materials Science from U. C. Berkeley in 1979, U.S.A. His Excellency, worked as a senior researcher in Materials Science Centre in Tsing-Hua University for several years and a faculty member as senior professor in Department of Physics, Tamkang University. Professor Lin published several hundred referred journal publications and held distinctive position in university. Professor I-Nan Lin supervised several PhD and Postdoc candidates around the world. Professor I-Nan Lin remained researching in the areas of developing high conductivity diamond films, composite materials and the deep-rooted studies of the transmission microscopy of materials. 